Mathematical Research Letters 10, 483-492 (2003)

\title{
A SYMPLECTIC CASE OF ARTIN'S CONJECTURE
}

\author{
Kimball Martin
}

\section{Introduction}

Here we will prove a new 4-dimensional symplectic case of Artin's conjecture. Let us first recall Artin's conjecture. Let $G$ be the Galois group of a finite Galois extension of number fields. Let $\rho$ be a representation of $G$ over $\mathbb{C}$. In this context one can associate to $\rho$ a meromorphic function $L(s, \rho)$ called the Artin $L$-function associated to $\rho$.

Artin's Conjecture. If $\rho$ does not contain the trivial representation, then $L(s, \rho)$ is entire.

An equivalent form of this conjecture is the following. If $\rho$ is irreducible and non-trivial, then $L(s, \rho)$ is entire. Artin proved this conjecture in the case where $\rho$ is 1-dimensional using his reciprocity law together with a result of Hecke. As $L$-functions are inductive, this proved the conjecture if $\rho$ is monomial, i.e. induced from a degree one representation of some subgroup.

Now consider the case where $\rho$ is 2-dimensional. Let $\bar{\rho}: G \rightarrow \mathrm{PGL}_{2}(\mathbb{C})$ be the composition of $\rho: G \rightarrow \mathrm{GL}_{2}(\mathbb{C})$ with the natural projection of $\mathrm{GL}_{2}(\mathbb{C})$ to $\mathrm{PGL}_{2}(\mathbb{C})$. Let $\bar{G}$ denote the image of $G$ under $\bar{\rho}$. So $\bar{G}$ is a finite subgroup of $\mathrm{PGL}_{2}(\mathbb{C})$, which is isomorphic to $\mathrm{SO}_{3}(\mathbb{C})$. The only finite subgroups of $\mathrm{SO}_{3}(\mathbb{C})$ are cyclic, dihedral, tetrahedral (isomorphic to $A_{4}$ ), octahedral (isomorphic to $S_{4}$ ) and icosahedral (isomorphic to $A_{5}$ ). We thus classify $\rho$ according the isomorphism type of $\bar{G}$. If $\bar{G}$ is cyclic or dihedral, then $\rho$ is reducible or monomial and $L(s, \rho)$ is entire by Artin's result. Much later, Langlands applied to this problem the theory of automorphic representations, which also have associated $L$-functions. For cuspidal automorphic representations of $\mathrm{GL}_{n}$ the associated $L$-function is known to be entire [Ja]. Both automorphic and Artin $L$-functions can be written as Euler products of local factors $L(s, \pi)=\prod_{v} L\left(s, \pi_{v}\right)$ and $L(s, \rho)=\prod_{v} L\left(s, \rho_{v}\right)$. Langlands formulated the following amazing conjecture [La1].

Strong Artin Conjecture. Let $G$ be the Galois group of an extension $K / F$ of number fields. Let $\rho$ be an $n$-dimensional complex representation of $G$. There exists an automorphic representation $\pi$ of $\mathrm{GL}_{n}\left(\mathbb{A}_{F}\right)$, such that the L-functions agree almost everywhere, i.e. except at a finite number of places $v, L\left(s, \rho_{v}\right)=$ $L\left(s, \pi_{v}\right)$. Moreover, if $\rho$ is irreducible, then $\pi$ is cuspidal.

Received January 8, 2003. 
We say $\rho$ is modular if the strong Artin conjecture holds for $\rho$. Furthermore, if $\pi$ is as in the conjecture, we will say $\rho$ corresponds to $\pi$ and write $\rho \leftrightarrow \pi$ or $\pi \leftrightarrow \rho$.

The strong Artin (also called Langlands' reciprocity) conjecture is really a stronger statement than Artin's conjecture. For example, nilpotent and supersolvable groups are monomial and hence their representations $\rho$ satisfy Artin's conjecture. Arthur and Clozel recently proved ([AC]) that if $\rho$ has nilpotent image, $\rho$ is also modular. However it has not yet been shown that all $\rho$ with supersolvable image must be modular.

Langlands proved the strong Artin conjecture, and hence Artin's conjecture, in the tetrahedral case [La2], i.e. when $\bar{G} \simeq A_{4}$ (and $\operatorname{dim} \rho=2$ ). In his proof, he used three important elements: the symmetric square lift from $\mathrm{GL}_{2}$ to $\mathrm{GL}_{3}$ of Gelbart and Jacquet [GJ], normal cubic base change on $\mathrm{GL}_{2}$ developed by him in [La2], and the structure of the group $A_{4}$. After non-normal cubic base change was proven by Jacquet, Piatetski-Shapiro and Shalika [JPSS], Tunnell used it to extend Langlands' argument and prove the strong Artin conjecture for the octahedral case $\left(\bar{G} \simeq S_{4}\right)$ [Tu]. This completed the solvable case in dimension 2 . The non-solvable (icosahedral) case is much more difficult. However, partial but outstanding progress has been made recently in this case by Buzzard, Dickinson, Shepherd-Barron and Taylor [BDST]. For modular 2-dimensional $\rho$, the corresponding symmetric $m$-th power representations for $m \leq 4$ are also modular by the work of Kim and Shahidi [KS], [Ki].

There are not many other cases of Artin's conjecture known in higher dimensions. Progress has been primarily made only for essentially self-dual (i.e., orthogonal or symplectic) representations. Let $\rho$ be an essentially self-dual irreducible complex $n$-dimensional representation of $G$ with solvable image. If $n$ is odd, then $\rho$ maps into $\mathrm{GO}_{n}(\mathbb{C})$ and $\rho$ is monomial [Ra]. Therefore in the odd-dimensional case Artin's conjecture (but not strong Artin) is known. If $n$ is even, $\rho$ has image either in $\mathrm{GO}_{n}(\mathbb{C})$ or $\operatorname{GSp}_{n}(\mathbb{C})$. In the case $\rho: G \rightarrow \mathrm{GO}_{4}(\mathbb{C})$ has solvable image, then Ramakrishnan recently showed $\rho$ satisfies the strong Artin conjecture by proving the modularity of the Asai representation [Ra].

Here we make progress in the case $\rho$ has image in $\operatorname{GSp}_{4}(\mathbb{C})$. We are able to exhibit another group whose 4-dimensional representations can be proven modular in a manner similar to Langlands' tetrahedral argument. Consider $\rho: G \rightarrow \operatorname{GSp}_{4}(\mathbb{C})$. Then $\bar{G} \subseteq \mathrm{PGSp}_{4}(\mathbb{C})=\mathrm{GSp}_{4}(\mathbb{C}) / \mathbb{C}^{*}$, which is isomorphic to $\mathrm{SO}_{5}(\mathbb{C})$. The finite subgroups of $\mathrm{PGSp}_{4}(\mathbb{C})$ have been classified in $[\mathrm{Mi}]$ and $[\mathrm{CM}]$. Let $E_{2^{4}}$ denote the elementary abelian group of order $2^{4}$ and $C_{5}$ the cyclic group of order 5 . Then there is a semidirect product $E_{2^{4}} \rtimes C_{5}$ with $C_{5}$ acting fixed point freely on $E_{2^{4}}$ contained inside $\operatorname{PGSp}_{4}(\mathbb{C})$. If $\bar{G} \simeq E_{2^{4}} \rtimes C_{5}$, we show $\rho$ is modular. Our proof uses the recent construction of Kim [Ki] of the exterior square $\Lambda^{2}(\pi)$ of an (isobaric) automorphic representation $\pi$ of $\mathrm{GL}_{4}$, which agrees locally almost everywhere with an automorphic representation of $\mathrm{GL}_{6}$. We also crucially use normal quintic base change ([AC]) and the structure of our group 
$E_{2^{4}} \rtimes C_{5}$. An investigation of the other subgroups of $\mathrm{PGSp}_{4}(\mathbb{C})$ will appear in the author's thesis. We now state the main result precisely.

Theorem 1. Let $L / F$ be a Galois extension of number fields and $\rho$ be an irreducible 4-dimensional representation of $G=\operatorname{Gal}(L / F)$ into $\operatorname{GSp}_{4}(\mathbb{C})$. Suppose $\bar{G}=\operatorname{Im}(\bar{\rho}) \simeq E_{2^{4}} \rtimes C_{5}$. Then $\rho$ is modular.

One pre-image of $E_{2^{4}} \rtimes C_{5}$ inside $\operatorname{Sp}_{4}(\mathbb{C})$ is $G=E_{2^{5}} \rtimes C_{5}$, where $E_{2^{5}}$ is the extraspecial group of order $2^{5}$ isomorphic to the central product of $Q_{8}$ and $D_{8}$ with identified centers (see [Do] for definitions). Consider an irreducible 4dimensional complex representation $\rho$ of $G$. As $G$ has no subgroups of index 2 or $4, \rho$ is primitive, i.e. not induced. Thus Artin's conjecture for $\rho$ does not follow from previous results.

We know that examples of $E_{2^{5}} \rtimes C_{5}$ extensions of $\mathbb{Q}$ exist by Shafarevich's Theorem ([NSW]) because $E_{2^{5}} \rtimes C_{5}$ is solvable. Though Shafarevich's proof is non-constructive, we can illustrate how to construct such an extension in our case. Let $\alpha_{i}=\zeta_{11}^{i}+\zeta_{11}^{-i}$, where $\zeta_{11}$ is a complex 11-th root of unity. Let $E$ be the cyclic Galois extension $\mathbb{Q}\left(\alpha_{1}\right)$ of $\mathbb{Q}$ of degree 5 . It is known how to construct $Q_{8}$ and $D_{8}$ extensions of a number field ([JLY]). Let $K=E(\sqrt{1+A+B+A B})$ and $M=E\left(\sqrt{\alpha_{1}+i \alpha_{2}}, \sqrt{\alpha_{2}+\alpha_{4}+4}\right)$, where $A=\left(3+\alpha_{5}\right)^{-1 / 2}$ and $B=(1+$ $\left.\alpha_{1}^{2}+\alpha_{1}^{2} \alpha_{3}^{2}\right)^{-1 / 2}$. Then $\operatorname{Gal}(K / E) \simeq Q_{8}$ and $\operatorname{Gal}(M / E) \simeq D_{8}$. The compositum $K M$ has three normal (over $E$ ) subextensions of index 2 . Let $L / E$ be the one corresponding to the central product of $Q_{8}$ with $D_{8}$. Then $L / \mathbb{Q}$ is Galois with Galois group $E_{2^{5}} \rtimes C_{5}$.

Before we go on, let us be a little more explicit about the similarities between Langlands' tetrahedral case and Theorem 1. We will first outline Langlands' argument. Let $\rho$ be a representation of $G$ into $\mathrm{GL}_{2}(\mathbb{C})$ with image $A_{4}$. Then $G$ has a normal subgroup $H$ of index 3 . Then $H$ is dihedral. All representations of dihedral groups are modular ([La2]), so $\rho_{H}$ is modular. Let $\Pi$ be an automorphic representation corresponding to $\rho_{H}$. Normal cubic base change for $\mathrm{GL}_{2}$ tell us that there are three representations $\pi_{i}$ whose base change $\pi_{i, H}$ (the automorphic version of restricting a representation) to $H$ is $\Pi$. So we think one of these $\pi_{i}$ should correspond to $\rho$. Using the determinant of $\rho$, Langlands proved there is a unique $\pi$ among the $\pi_{i}$ 's with its central character $\omega_{\pi}$ corresponding to $\operatorname{det}(\rho)$, such that $\operatorname{Sym}^{2}(\rho) \leftrightarrow \operatorname{Sym}^{2}(\pi)$. Langlands then showed that if $\rho_{H} \leftrightarrow \pi_{H}$ and $\operatorname{Sym}^{2}(\rho) \leftrightarrow \operatorname{Sym}^{2}(\pi)$, but $\rho$ does not correspond to $\pi$, then $\bar{G} \simeq A_{4}$ must have an element of order 6 . But $A_{4}$ has no elements of order 6 , therefore $\rho$ must correspond to $\pi$, i.e. $\rho$ is modular.

The basic approach to proving Theorem 1 is similar to the tetrahedral argument, but with several differences. The first difficulty encountered in our case is that the determinant alone does not give enough information to choose the appropriate $\pi$. More precisely, suppose $\rho$ is a representation of $G$ into $\operatorname{GSp}_{4}(\mathbb{C})$ with projective image $E_{2^{4}} \rtimes C_{5}$. Then $G$ has a normal subgroup $H$ of index 5 . Since $H$ is nilpotent, $\rho_{H}$ is modular. Say $\rho_{H} \leftrightarrow \Pi$. Normal quintic base change tells us that there are five representations $\pi_{i}$ whose base change $\pi_{i, H}$ to $H$ equals 
$\Pi$. Using the fact that $\Lambda^{2}(\rho)$ has an invariant line, we are able to pick a unique $\pi$ from the $\pi_{i}$ 's such that $\Lambda^{2}(\rho) \leftrightarrow \Lambda^{2}(\pi)$. Then we deduce either $\rho \leftrightarrow \pi$ or $\bar{G}$ has an element of order 10, which it does not. Therefore $\rho$ must be modular.

In fact, Langlands' conjectures actually predict $\rho$ is modular of symplectic type, i.e $\rho$ corresponds to an automorphic representation of $\operatorname{GSp}_{4}\left(\mathbb{A}_{F}\right)$. We discuss this briefly in the final section.

\section{Preliminaries}

In this section, we will review the theories of $L$-functions and base change. Those already familiar with them may wish to skip to the proof in the next section.

First we will begin with some notation. Let $F$ be a number field and $L$ be a finite Galois extension of $F$ with Galois group $G$. Let $\rho$ be a representation of $G$ into $\mathrm{GL}_{n}(\mathbb{C})$. We will refer to restriction and induction of representations with the corresponding fields. More precisely, let $E$ be a subextension of $L / F$ and $H=\operatorname{Gal}(L / E)$. Then $\rho_{E}$ denotes the restriction $\rho_{H}$ of $\rho$ to the subgroup $H$. For a representation $\sigma$ of $H, I_{E}^{F} \sigma$ denotes the induced representation $I_{H}^{G} \sigma$ of $\sigma$ from $H$ to $G$. We will use $\mathfrak{N}_{E / F}$ to denote the norm map from $E$ to $F$.

Let $v$ be a place of $F$ and $\mathrm{Fr}_{v}$ be the corresponding Frobenius class. Denote by $q_{v}$ the size of the residue field $\mathcal{O}_{F} / v$. Recall the Artin $L$-function is defined on some right-half plane by $L(s, \rho)=\prod_{v} L\left(s, \rho_{v}\right)$ where the product is over all places $v$ of $F$ and if $v$ is unramified,

$$
L\left(s, \rho_{v}\right)=\frac{1}{\operatorname{det}\left(1-\rho\left(\operatorname{Fr}_{v}\right) q_{v}^{-s}\right)} .
$$

Brauer showed for any $\rho, L(s, \rho)$ extends to a meromorphic function on all of $\mathbb{C}$. In the case $\rho$ is the trivial representation 1 , the $L$-function is the Dedekind zeta function $\zeta_{F}(s)$ of $F$. For a complete definition of Artin $L$-functions, see [Ro], $[\mathrm{Ne}]$ or $[\mathrm{Ma}]$.

One can also define $L$-functions for automorphic representations. Let $\mathbb{A}_{F}$ be the adeles of the number field $F$.

Theorem 2.2 ([Ja]). Let $\pi$ be an automorphic representation of $\mathrm{GL}_{n}\left(\mathbb{A}_{F}\right)$. Then we can associate local factors $L\left(s, \pi_{v}\right)$ to each $v$ and define a holomorphic $L$-function $L(s, \pi)=\prod L\left(s, \pi_{v}\right)$ in some right-half plane. Moreover $L(s, \pi)$ extends to an meromorphic function on $\mathbb{C}$, which is actually entire if $\pi$ is cuspidal and non-trivial.

For more information on automorphic representations and their $L$-functions, see $[\mathrm{Ge} 1],[\mathrm{Kn}]$, or $[\mathrm{Ge} 2]$. The strong Artin conjecture asserts that given a Galois representation $\rho$ as before, there exists an automorphic representation $\pi$ corresponding to $\rho$ (see Introduction). If $\rho \leftrightarrow \pi$ and $\rho$ is irreducible, then the following result implies $\pi$ is cuspidal. 
Theorem 2.3 ([JS1]). Let $\pi$ be an automorphic representation of $\mathrm{GL}_{n}\left(\mathbb{A}_{F}\right)$ and $\check{\pi}$ its contragredient. Then $L(s, \pi \times \check{\pi})$ has a simple pole at $s=1$ if and only if $\pi$ is cuspidal.

For if $\rho$ is irreducible and $\check{\rho}$ its contragredient, then $L(s, \rho \otimes \check{\rho})$ has a simple pole at $s=1$. Moreover if $\rho \leftrightarrow \pi$, then $L(s, \rho \otimes \check{\rho})$ and $L(s, \pi \times \check{\pi})$ agree almost everywhere locally. So by Theorem $2.3, \pi$ is indeed cuspidal.

Now to show that the strong Artin conjecture actually implies Artin's conjecture we only need the following standard fact.

Proposition 2.1. If $\pi$ is cuspidal and $L\left(s, \pi_{v}\right)=L\left(s, \rho_{v}\right)$ for almost all $v$, then in fact $L(s, \pi)=L(s, \rho)$.

So if $\rho$ is irreducible and non-trivial and corresponds to $\rho$, then by Theorem $2.3 \rho$ is cuspidal (and non-trivial). The proposition tells us that in fact $L(s, \pi)=$ $L(s, \rho)$. By Theorem 2.2 we know that $L(s, \pi)$ is entire, i.e. $L(s, \rho)$ is entire, i.e. Artin's conjecture is true for $\rho$.

If $\pi$ and $\pi^{\prime}$ are cuspidal representations of $\mathrm{GL}_{m}\left(\mathbb{A}_{F}\right)$ and $\mathrm{GL}_{n}\left(\mathbb{A}_{F}\right)$ respectively, then one can form their isobaric sum $\pi \boxplus \pi^{\prime}$ which is an automorphic representation of $\mathrm{GL}_{m+n}\left(\mathbb{A}_{F}\right)$ [JS1]. If $\rho \leftrightarrow \pi$ and $\rho^{\prime} \leftrightarrow \pi^{\prime}$, then $\rho \oplus \rho^{\prime} \leftrightarrow \pi \boxplus \pi^{\prime}$. So every automorphic representation which corresponds to a Galois representation will be isobaric, i.e. a finite isobaric sum of cuspidal representations.

A powerful tool to prove the strong Artin conjecture in certain instances is the theory of base change. Base change is an operation on automorphic representations which corresponds to the restriction of Galois representations. We now list the important properties of base change that we need.

Theorem 2.4 ([AC]). Let $L / F$ be a Galois extension of number fields. Let $E / F$ be a normal cyclic subextension of prime degree. For each isobaric representation $\pi$ of $\mathrm{GL}_{n}\left(\mathbb{A}_{F}\right)$, there exists a unique automorphic representation of $\mathrm{GL}_{n}\left(\mathbb{A}_{E}\right)$ called the base change of $\pi$ to $E$ and denoted by $\pi_{E}$ such that:

(i) (descent) a cuspidal representation $\Pi$ of $\mathrm{GL}_{n}\left(\mathbb{A}_{E}\right)$ is the base change $\pi_{E}$ of some $\pi$ if and only if $\Pi$ is Galois invariant (in particular, if $\Pi \leftrightarrow \rho_{E}$ where $\rho$ is some representation of $\operatorname{Gal}(L / F))$;

(ii) if $\pi^{\prime}$ is also an isobaric representation of $\mathrm{GL}_{n}\left(\mathbb{A}_{F}\right)$ then $\pi_{E}=\pi_{E}^{\prime}$ if and only if $\pi^{\prime}=\pi \otimes \delta$ for some idele class character $\delta$ of $F^{*} \mathfrak{N}_{E / F}\left(\mathbb{A}_{E}^{*}\right) \backslash \mathbb{A}_{F}^{*} \simeq$ $\operatorname{Gal}(E / F)$;

(iii) (compatibility with reciprocity) if $\rho$ is a representation of $\operatorname{Gal}(L / F), \rho \leftrightarrow \pi$ and $\rho_{E}$ is modular, then $\rho_{E} \leftrightarrow \pi_{E}$; and

(iv) (compatibility with twisting) if $\chi$ is an idele class character of $F$ and $\chi_{E}=$ $\chi \circ N_{E / F}$, then

$$
(\pi \otimes \chi)_{E}=\pi_{E} \otimes \chi_{E} .
$$

The complementary construction to base change is automorphic induction, which corresponds to induction of Galois representations. 
Theorem 2.5 ([AC], $[\mathrm{HH}])$. Let $L / F$ be a Galois extension of number fields. Let $E / F$ be a normal cyclic subextension of prime degree. Let $\rho$ be a complex representation of $\operatorname{Gal}(L / E)$ and suppose $\rho \leftrightarrow \pi$, for some automorphic representation $\pi$. Then there exists an induced automorphic representation, denoted $I_{E}^{F} \pi$ such that $I_{E}^{F} \rho \leftrightarrow I_{E}^{F} \pi$.

\section{Proof of Theorem 1}

Let $L / F$ be a (finite) Galois extension of number fields with Galois group $G$. Suppose $\rho$ is an (injective) representation of $G$ into $\mathrm{GSp}_{4}(\mathbb{C})$ such that $\bar{G} \simeq E_{2^{4}} \rtimes C_{5}$. Let $E$ be the normal quintic subextension of $L / F$ corresponding to the pre-image of $E_{2^{4}}$.

Lemma 3.1. The representations $\rho_{E}$ and $\Lambda^{2}(\rho)$ are modular.

Proof. As $\operatorname{Gal}(L / E)$ is a cyclic central extension of a 2-group, it is a direct product of a 2-group $P_{2}$ with a cyclic group $C$ of odd order. Therefore $\operatorname{Gal}(L / E)$ is nilpotent. By a theorem of Arthur and Clozel, all representations of nilpotent groups are modular $[\mathrm{AC}]$. In particular $\rho_{E}$ is modular.

Since $\rho$ is of symplectic type, $\Lambda^{2}(\rho)$ has an invariant line. Write $\Lambda^{2}(\rho)=\nu \oplus r$ where $\nu$ is 1-dimensional and $r$ is 5 -dimensional. Note $r$ is irreducible because it factors through $E_{2^{4}} \rtimes C_{5}$, which only has 1- and 5- dimensional irreducible representations. We claim $r$ is induced from $E$. As $P_{2}$ is a 2-group, every irreducible representation of $P_{2}$ has dimension $2^{j}$ for some $j$. Therefore the same is true for $\operatorname{Gal}(L / E) \simeq P_{2} \times C$. Hence in the decomposition of $r_{E}$ into its irreducible components, we must have a 1-dimensional representation $\lambda$. In particular, $r=I_{E}^{F} \lambda$. Since $E$ is a normal subextension $r$ is modular by Theorem 2.5 , whence $\Lambda^{2}(\rho)$ is also.

Let us say $\rho_{E} \leftrightarrow \Pi$. We claim $\rho_{E}$ is irreducible. Indeed, $\rho$ irreducible implies that $\operatorname{Gal}(E / F) \simeq C_{5}$ acts transitively on the irreducible components of $\rho_{E}$. This action has order dividing 5 . Thus if there is more than one irreducible component of $\rho_{E}$, there must be five or a multiple thereof. However $\operatorname{dim} \rho_{E}=4$, so that is impossible. Then by Theorem $2.3, \Pi$ is cuspidal.

We can apply Theorem 2.4(i) to get an automorphic representation $\pi_{0}$ of $\mathrm{GL}_{4}\left(\mathbb{A}_{F}\right)$ whose base change $\pi_{0, E}$ corresponds to $\rho_{E}$. Let $\delta=\delta_{E / F}$ be a nontrivial idele class character of $F^{*} \mathfrak{N}_{E / F}\left(\mathbb{A}_{E}^{*}\right) \backslash \mathbb{A}_{F}^{*} \simeq \operatorname{Gal}(E / F) \simeq C_{5}$. Let $\pi_{i}=$ $\pi_{0} \otimes \delta^{i}$ for $i=1,2, \ldots, 4$. Since $\delta_{E}=\mathbf{1}$, all the $\pi_{i}$ 's base change to $\pi_{i, E} \simeq$ $\left(\pi_{0} \otimes \delta\right)_{E} \simeq \pi_{0, E} \simeq \Pi$ by Theorem 2.4(iv). In fact, part (ii) of the same theorem tells us that these are all the cuspidal representations of $\mathrm{GL}_{4}\left(\mathbb{A}_{F}\right)$ whose base change to $E$ is $\Pi$.

Lemma 3.2. There is a unique $i \in\{0,1,2, \ldots, 4\}$ such that $\Lambda^{2}\left(\pi_{i}\right) \leftrightarrow \Lambda^{2}(\rho)$.

Proof. All the representations $\Lambda^{2}\left(\pi_{i}\right)$ base change to $\Lambda^{2}\left(\pi_{0} \otimes \delta^{i}\right)_{E}=\Lambda^{2}\left(\pi_{0}\right)_{E}$ by Theorem 2.4(iv). They are all distinct because they have distinct central characters $\omega_{\Lambda^{2}\left(\pi_{i}\right)}=\omega_{\Lambda^{2}\left(\pi_{0}\right)} \delta^{2 i}$. 
Theorem 2.4(ii) then yields that the $\Lambda^{2}\left(\pi_{i}\right)$ are the only representations which base change to $\Lambda^{2}\left(\pi_{0}\right)_{E}$. But by part (iii) of this theorem, the automorphic representation on $\mathrm{GL}_{6}\left(\mathbb{A}_{F}\right)$ which corresponds to $\Lambda^{2}(\rho)$ must also base change to $\Lambda^{2}\left(\pi_{0}\right)_{E}$. Thus for some $i, \Lambda^{2}\left(\pi_{i}\right) \leftrightarrow \Lambda^{2}(\rho)$.

Denote the $\pi_{i}$ of the lemma by $\pi$. We claim now that in fact $\rho \leftrightarrow \pi$. It will suffice to show for all unramified places that $\rho_{v} \leftrightarrow \pi_{v}$. Say $\rho_{v}$ has Frobenius eigenvalues $\{a, b, c, d\}$ and $\pi_{v}$ has Satake parameters $\{e, f, g, h\}$. We want to show $\{a, b, c, d\}=\{e, f, g, h\}$. Let $D$ be a diagonal element of $\mathrm{GL}_{4}$. Then $\Lambda^{2}(D)=1$ if and only if $D= \pm I$. Hence $\Lambda^{2}\left(\rho_{v}\right) \leftrightarrow \Lambda^{2}\left(\pi_{v}\right)$ implies

$$
\{a, b, c, d\}= \pm\{e, f, g, h\} .
$$

If they are equal, we are done. Assume therefore

$$
\{a, b, c, d\}=-\{e, f, g, h\} .
$$

Now we can use base change to $E$. In our group $\bar{G}$ any element raised to the 5 th power lies inside $E_{2^{4}}$ (see Lemma 3.3 below). Thus any element of $G$ raised to the 5th power lies inside $\operatorname{Gal}(L / E)$, the pre-image of $E_{2^{4}}$. In particular $F r_{v}^{5} \in \mathcal{O}_{E_{w}}$, where $w$ is a prime of $E$ above $v$. Then $\rho_{v, E} \leftrightarrow \pi_{v, E}$ implies $\left\{a^{5}, b^{5}, c^{5}, d^{5}\right\}=\left\{e^{5}, f^{5}, g^{5}, h^{5}\right\}$. By our assumption we have,

$$
\left\{a^{5}, b^{5}, c^{5}, d^{5}\right\}=\left\{-a^{5},-b^{5},-c^{5},-d^{5}\right\} .
$$

Without loss of generality, assume $a^{5}=-b^{5}$ and $c^{5}=-d^{5}$. Then either $b=-\zeta_{5} a$ or $d=-\zeta_{5} c$, for otherwise $a=-b, c=-d$ which would imply $\{a, b, c, d\}=$ $\{e, f, g, h\}$. Let us say

$$
b=-\zeta_{5} a .
$$

Then

$$
\rho\left(F r_{v}\right) \sim\left(\begin{array}{cccc}
a & 0 & 0 & 0 \\
0 & -\zeta_{5} a & 0 & 0 \\
0 & 0 & c & 0 \\
0 & 0 & 0 & d
\end{array}\right)
$$

so

$$
\bar{\rho}\left(F r_{v}\right) \sim\left(\begin{array}{cccc}
1 & 0 & 0 & 0 \\
0 & -\zeta_{5} & 0 & 0 \\
0 & 0 & c / a & 0 \\
0 & 0 & 0 & d / a
\end{array}\right),
$$

is an element of order divisible by 10 in $\bar{G}=\operatorname{Im}(\bar{\rho}) \subseteq \operatorname{PSp}_{4}(\mathbb{C})$. But $\bar{G}$ has no elements of order 10 by Lemma 3.3 below, so $\rho$ is modular by contradiction.

Lemma 3.3. Every element $g \in E_{2^{4}} \rtimes C_{5}$ has order 5 except for the elements in the normal subgroup $E_{2^{4}}$, which have order 1 or 2. 
Proof. Let $g \in E_{2^{4}} \rtimes C_{5}$ such that $g \notin E_{2^{4}}$. We can write $g=a z$ where $a \in E_{2^{4}}$ and $z \in C_{5}, z \neq 1$. We claim $g^{5}$ commutes with $z$. Write

$$
\begin{aligned}
g^{5} & =(a z)(a z)(a z)(a z)(a z) \\
& =a\left(z a z^{-1}\right)\left(z^{2} a z^{-2}\right)\left(z^{3} a z^{-3}\right)\left(z^{4} a z^{-4}\right) .
\end{aligned}
$$

We also have

$$
\begin{aligned}
z g^{5} z^{-1} & =(z a)(z a)(z a)(z a)(z a) \\
& =\left(z a z^{-1}\right)\left(z^{2} a z^{-2}\right)\left(z^{3} a z^{-3}\right)\left(z^{4} a z^{-4}\right) a .
\end{aligned}
$$

Each $z^{j} a z^{-j}$ lies in the normal abelian subgroup $E_{2^{4}}$ and therefore the $z^{j} a z^{-j}$ 's commute. Thus we can rearrange the terms in (3.10) to get (3.8) and we have $z g^{5} z^{-1}=g^{5}$.

Now, since each term in (3.8) lies in $E_{2^{4}}$, then $g^{5} \in E_{2^{4}}$ also. But the action of $C_{5}$ on $E_{2^{4}}$ fixes only the identity. Thus $g^{5}=1$.

\section{Transfer to $\mathrm{GSp}_{4}$}

As before, consider a Galois group $G$ of an extension of number fields $L / F$ and an irreducible 4-dimensional representation $\rho$ of $G$ into $\operatorname{GSp}_{4}(\mathbb{C})$. Suppose that $\rho$ is modular, i.e. $\rho$ corresponds to some cuspidal representation $\pi$ of $\mathrm{GL}_{4}\left(\mathbb{A}_{F}\right)$. The fact that $\operatorname{Im}(\rho) \subseteq \mathrm{GSp}_{4}(\mathbb{C})$ implies that $L\left(s, \Lambda^{2}(\rho) \otimes \nu^{-1}\right)$ has a simple pole at $s=1$ for a suitable 1-dimensional representation $\nu$ of $G$ (the "polarization").

This implies that the corresponding automorphic $L$-function $L\left(s, \pi ; \Lambda^{2} \otimes \nu^{-1}\right)$ admits a pole at $s=1$. An unpublished theorem of Jacquet, Piatetski-Shapiro and Shalika says that, because of this pole, $\pi$ transfers to a generic irreducible cuspidal automorphic representation $\Pi$ of $\operatorname{GSp}_{4}\left(\mathbb{A}_{F}\right)$ with central character $\nu$ such that,

$$
L^{S}(s, \Pi)=L^{S}(s, \pi),
$$

for any finite set of primes $S$ outside of which $\pi$ is unramified. Here the $L$ function on the left is the degree $4 L$-function of $\Pi$ studied in [PS]; and if $L(s)=\prod_{v} L_{v}(s)$ is an Euler product, then $L^{S}(s)$ denotes the incomplete $L$ function $\prod_{v \notin S} L_{v}(s)$. Thus $\rho$ in fact corresponds to the cuspidal representation $\Pi$ of $\mathrm{GSp}_{4}\left(\mathbb{A}_{F}\right)$, i.e. $\rho$ is modular of symplectic type as predicted by Langlands.

However we are not stressing this here because this theorem of Jacquet, Piatetski-Shapiro and Shalika remains unpublished. We hope to go into more detail in the thesis. The key point is that $\mathrm{GL}_{4}$ maps into the connected component of $\mathrm{GO}_{6}$, and $\pi$ gives rise to a cuspidal automorphic representation $\pi^{\prime}$ of $\mathrm{GO}_{6}\left(\mathbb{A}_{F}\right)^{0}$. The desired $\Pi$ is obtained by the theta correspondence. The obstruction to this transfer is the residue of the pole of $L\left(s, \pi ; \Lambda^{2} \otimes \nu^{-1}\right)$ at $s=1$ (see [JS2]). Finally the ongoing work of J. Arthur will give another proof, using the trace formula, of the existence of $\Pi$ and other members of its packet (see $[\mathrm{Ar}]$ for his program). 


\section{Acknowledgements}

The author would like to foremost thank his advisor, Dinakar Ramakrishnan, for suggestions and guidance throughout this work. He would also like to thank David Wales and Michael Aschbacher for their useful words about symplectic groups. The author is grateful to Jim Cogdell, David Whitehouse and the referee for comments that led to several minor improvements and corrections. Finally, thanks go to the GAP Group for their wonderful package as many group and character computations were done in the initial stages of this work using [GAP].

\section{References}

[AC] J. Arthur, L. Clozel, Simple algebras, base change, and the advanced theory of the trace formula, Annals of Mathematics Studies, 120. Princeton University Press, Princeton, NJ, 1989.

[Ar] J. Arthur, Automorphic representations of $G S p(4)$, to appear in Contributions to Automorphic Forms, Geometry and Number Theory (Shalikafest 2002).

[BDST] K. Buzzard, M. Dickinson, N. I. Shepherd-Barron, R. L. Taylor, On icosahedral Artin representations, Duke Math. J. 109 (2001), 283-318.

[CM] M. Cazzola, L. Di Martino, (2,3)-generation of $P S p(4, q), q=p^{n}, p \neq 2,3$, Results Math. 23 (1993), 221-232.

[Do] L. Dornhoff, Group representation theory. Part A: Ordinary representation theory, Pure and Applied Mathematics, 7. Marcel Dekker, Inc., New York, 1971.

[GAP] The GAP Group, GAP — Groups, Algorithms, and Programming, Version 4.3, 2002: http://www.gap-system.org

[Ge1] S. Gelbart, An elementary introduction to the Langlands program, Bull. Amer. Math. Soc. (N.S.) 10 (1984), 177-219.

[Ge2] - Automorphic forms on adèle groups, Annals of Mathematics Studies, 83. Princeton University Press, Princeton, NJ, 1975.

[GJ] S. Gelbart, H. Jacquet, A relation between automorphic representations of $G L(2)$ and GL(3), Ann. Sci. École Norm. Sup. (4) 11 (1978), 471-542.

[HH] G. Henniart, R. Herb, Automorphic induction for $G L(n)$ (over local non-archimedean fields), Duke Math. J. 78 (1995), 131-192.

[Ja] H. Jacquet, Principal L-functions of the linear group, Proc. Sympos. Pure Math. 33 (1979), 63-86.

[JPSS] H. Jacquet, I. I. Piatetski-Shapiro, J. Shalika, Automorphic forms on GL(3). II, Ann. of Math. (2) 109 (1979), 213-258.

[JS1] H. Jacquet, J. Shalika, On Euler products and the classification of automorphic forms. I, II, Amer. J. Math. 103 (1981), 499-558, 777-815.

[JS2] - Exterior square L-functions, Automorphic forms, Shimura varieties, and $L$ functions, Vol. II (Ann Arbor, MI, 1988), 143-226, Perspect. Math., 11, Academic Press, Boston, MA, 1990.

[JLY] C. Jensen, A. Ledet, N. Yui, Geometric polynomials: constructive aspects of the inverse Galois problem, MSRI Pub. 45 (2002).

[Ki] H. Kim, Functoriality for the exterior square of $G L_{4}$ and the symmetric fourth of $G L_{2}$, J. Amer. Math. Soc. 16 (2003), 139-183.

[KS] H. Kim, F. Shahidi, Functorial products for $G L_{2} \times G L_{3}$ and functorial symmetric cube for $G L_{2}$, C. R. Acad. Sci. Paris Sér. I Math. 331 (2000), 599-604.

[Kn] A. W. Knapp, Introduction to the Langlands program, Proc. Symp. Pure Math. 61 (1997), 245-302.

[La1] R. P. Langlands, Letter to André Weil from January, 1967: http://www.sunsite.ubc.ca/DigitalMathArchive/Langlands/functoriality.html 
[La2] , Base Change for GL(2), Annals of Mathematics Studies, 96. Princeton University Press, Princeton, NJ, 1980.

[Ma] J. Martinet, Character theory and Artin L-functions, Algebraic Number Fields: Lfunctions and Galois Properties, Academic Press, London, 1977.

[Mi] H.H. Mitchell, The subgroups of the quaternary abelian linear group, Trans. Amer. Math. Soc. 15 (1914), 379-396.

[Ne] J. Neukirch, Algebraic number theory. Translated from the 1992 German original and with a note by Norbert Schappacher. Grundlehren der Mathematischen Wissenschaften, 322. Springer-Verlag, Berlin, 1999.

[NSW] J. Neukirch, A. Schmidt, K. Wingberg, Cohomology of number fields, Grundlehren der Mathematischen Wissenschaften, 323. Springer-Verlag, Berlin, 2000.

[PS] I. I. Piatetski-Shapiro, L-functions for $\mathrm{GSp}_{4}$, Olga Taussky-Todd: in memoriam. Pacific J. Math. 1997, Special Issue, 259-275.

[Ra] D. Ramakrishnan, Modularity of solvable Artin representations of GO(4)-type, Int. Math. Res. Not. 2002, 1-54.

[Ro] J. Rogawski, Functoriality and the Artin conjecture, Proc. Symp. Pure Math. 61 (1997), 331-353.

[Tu] J. Tunnell, Artin's conjecture for representation of octahedral type, Bull. Amer. Math. Soc. 5 (1981), 173-175.

253-37 Caltech, Pasadena, CA 91125, U.S.A.

E-mail address: kimball@caltech.edu 